\title{
Aspectos Fonoaudiológicos na Atrofia de Múltiplos Sistemas(AMS)
}

\author{
Denise Botelho Knopp ${ }^{1}$, Henrique Ballalai Ferraz ${ }^{2}$
}

\section{RESUMO}

Recentemente, muitos estudos apontam a presença de disartria como um importante sinal no diagnóstico diferencial da Atrofia de Múltiplos Sistemas (AMS), outros estudos afirmam que o aparecimento de disartria e disfagia durante o primeiro ano de doença, em paciente com parkinsonismo de etiologia degenerativa, sugere fortemente 0 diagnóstico de AMS ou outras formas de parkinsonismo atípico. Neste artigo apresentamos a definição de disartrofonia, consideramos a contribuição fonoaudiológica no diagnóstico diferencial da AMS, e as mais recentes pesquisas no assunto.

Descritores: Atrofia de múltiplos sistemas, Síndrome parkinsoniana, Insuficiência autonômica, Disartria, Transtorno da fala, Transtorno da voz.

\section{SUMMARY}

Recently, many studies point dysarthria as an important signal for the diagnosis of Multisystem Atrophy (MSA). Dysarthria and dysphagia during the first year of illness, in patient with degenerative parkinsonism, strongly suggests the diagnosis of MSA or other forms of atypical parkinsonism. In this article we present the definition of dysartrophonia, and we show the speech pathology role in the diagnosis of the MSA, and also the most recent researches on this subject.

Key words: Multisystem atrophy, Parkinsonian syndrome, Autonomic insufficiency, dysarthria, voice disorders, speech disorders.

\section{Introdução}

Atrofia de múltiplos sistemas (AMS) é o termo usado para a unificação de entidades previamente distintas: a atrofia olivopontocerebelar, a síndrome de Shy-Drager, e a degeneração estriatonigral. $\mathrm{O}$ primeiro relato clínico desta entidade data de 1900, quando Dejèrine e Thomas descrevem dois pacientes com um distúrbio neurológico não-familiar caracterizado por sinais cerebelares, parkinsonismo e disautonomia, chamando esta entidade de atrofia olivopontocerebelar ${ }^{1}$. Sessenta anos depois, Shy e Drager descreveram dois casos com disautonomia e parkinsonismo, demonstrando perda neuronal na substância negra, corpo estriado e células da coluna intermediolateral, além dos locais descritos, anteriormente; (chamaram de síndrome de Shy-Drager) ${ }^{2}$. Finalmente, em 1969, Graham e Oppenheimer juntaram os três componentes clinicopatológicos sob o nome de atrofia de múltiplos sistemas, estabelecendo-a como uma entidade diagnóstica independente ${ }^{3}$.

Atualmente calcula-se que a prevalência da AMS seja de aproximadamente $3 / 100000$ na população com idade entre 50-99 anos, e é provavelmente a terceira causa mais freqüente de parkinsonismo degenerativo, superada apenas pela doença de Parkinson (DP) e pela Paralisia Supranuclear Progressiva (PSP). O diagnóstico definitivo somente é possível na avaliação anatomopatológica, com a presença das inclusões citoplasmáticas gliais, sugerindo que a alterações gliais tenham papel fundamental em sua etiopatogenia ${ }^{4}$. Entretanto, a etiologia definitiva desta entidade continua incerta, sendo sugerida, em alguns estudos, a participação de fatores ambientais ${ }^{5}$.

No inicio, o quadro clínico pode ser semelhante ao da DP, especialmente na forma estriatonigral, trazendo dificuldades diagnósticas para o neurologista. Durante a evolução, o aparecimento de manifestações pouco comuns na DP sugerem o diagnóstico de $\mathrm{AMS}^{6}$, em alguns casos, o diagnóstico diferencial com a DP ou outras síndromes Parkinson-plus pode levar muitos anos.

A presença de disartria é um importante sinal no diagnóstico diferencial da AMS, e segundo Wenning et al $(2000)^{7}$ cinco fatores podem ajudar na diferenciação entre a AMS e a DP: 1) pouca ou nenhma resposta à levodopa; 2) disartria e disfagia; 3)quedas recorrentes; 4)disfunção autonômica e 5)função cognitiva preservada. Quinn $(1989)^{8}$ ressalta que no início da doença, o diagnóstico diferencial da AMS pode ser difícil, porém a presença de palilalia e disartria precocemente, entre outros fatores, levaria a uma forte suspeita. Bassich et al $(1984)^{9} \mathrm{em}$ estudos com 10 pacientes com disfunção autonômica associada a distúrbio neurológico concluem que a 
presença de sintomas de fala pode auxiliar na diferenciação entre pacientes com disfunção autonômica pura daqueles com envolvimento central. De acordo com alguns autores a disartria e a disfagia são sintomas que ocorrem mais tardiamente do que os sintomas motores na $\mathrm{AMS}^{10}$. Por outro lado em 2001, o International Medical Workshop Covering Progressive Supranuclear Palsy, Multiple System Atrophy, and Cortico Basal Degeneration ${ }^{11}$ afirma que o aparecimento de disartria e disfagia durante o primeiro ano de doença, em paciente com parkinsonismo de etiologia degenerativa, sugere fortemente o diagnóstico de AMS ou outras formas de parkinsonismo atípico. Nesta mesma linha, Knopp et al $(2002)^{12}$ ao estudar 5 pacientes com o diagnóstico provável de AMS, observaam que havia comprometimento da fala já durante o primeiro ano de doença, em todos os pacientes da amostra.

\section{Definição Disartria/Disartrofonia}

A importância da voz e fala na sociedade moderna não pode ser subestimada, pois é o instrumento básico por meio do qual transmitimos pensamentos, desejos e emoções, projetamos nossa personalidade e influenciamos as pessoas. Portanto, a fala apresenta importância pessoal, social e econômica. Ramig \& Sherer(1992) ${ }^{13}$ afirmam que a deterioração progressiva da fala e a inabilidade em comunicar-se efetivamente com a família, amigos e empregados têm sido o impacto mais negativo na qualidade de vida, auto-estima e esperança para o futuro em pacientes parkisnonianos.

A articulação da fala é o processo no qual as estruturas da cavidade oral são movidas e modeladas para produzir configurações específicas e gerar, consequentemente, padrões acústicos da onda sonora. Padrões articulatórios específicos são determinados pelo tamanho e formato das cavidades, como também pela magnitude, movimento e constrições musculares ao longo do trato vocal ${ }^{14}$.

A disartrofonia, corresponde às alterações que resultam de distúrbios do controle muscular causados por lesões do sistema nervoso central ou periférico ${ }^{15}$. A disartrofonia pode ser classificada como hipocinética, atáxica, hipercinética, espástica e mista ${ }^{16}$. Para que se desenvolvam métodos eficazes de tratamento fonoaudiológico que melhorem a comunicação dos pacientes, se faz necessário, primeiramente, conhecer melhor quais são as alterações fonoaudiológicas que acompanham a doença.

\section{Disartrofonia na AMS}

Setenta e cinco a cem porcento dos portadores de parkinsonismo desenvolvem alterações de voz e fala que influenciam negativamente sua habilidade de comunicarse com a família e amigos e limitam suas oportunidades de emprego ${ }^{17,18}$. Uma abordagem eficiente de terapia para esta população ainda é pouco documentada, e além disso, a intervenção fonoaudiológica usualmente ocorre somente quando a doença já esta em estágio avançado. Nesta situação, o paciente já está tão debilitado que a única opção que resta é a de se utilizar os recursos de comunicação alternativa. Muitos pacientes permanecem meses ou anos com uma comunicação oral muito prejudicada diminuindo significativamente a qualidade de vida.
Em análise mais específica em pacientes com AMS, Wenning et al (1994) ${ }^{19}$ em um estudo com 100 casos, notou a presença de disatrofonia em quase todos os pacientes e relata que esta, freqüentemente não era uma disartria típica parkinsoniana. Kluin et al (1996) $)^{20}$ avaliaram 46 pacientes com AMS através de análise perceptivo auditiva e motora oral. Encontraram que $96 \%$ os pacientes apresentavam disartria mista na combinação dos componentes hipocinéticos, atáxicos e espásticos; sendo que $48 \%$ apresentou predomínio dos sintomas hipocinéticos, 35\% atáxico, $11 \%$ espástico, 2\% só apresentaram sintomas hipocinéticos e espásticos e $2 \%$ somente sintomas atáxicos. A presença de disartria mista com combinação dos componentes hipocinéticos, atáxicos e espásticos também é apontada por Rehman $(2001)^{21}$ e Knopp et al (2002)12 como uma das características da AMS.

Inicialmente, a disartrofonia da AMS era descrita separadamente para as suas três formas como na descrição de Duffy $(1995)^{22}$. Este, relata que na atrofia olivopontocerebelar pode-se encontrar uma disartria hipocinética semelhante a da doença de Parkinson, mas, geralmente, ocorre uma disartria atáxica caracterizada por imprecisão articulatória, pausa irregulares, distorção de vogais, excesso e ênfase na prosódia, prolongamento de sons, diminuição da velocidade de fala, e voz rouca e monótona. Na doença de Shy-Drager, por sua vez, encontra-se uma disartria mista hipocinética-espástica, com características de imprecisão articulatória, rouquidão, velocidade de fala lentificada, distorção de vogais, voz monótona, e alteração da ênfase da prosódia para excessiva ou reduzida. No estudo de Countryman et al $(1994)^{23}$ que avalia a efetifidade do método de tratamento vocal Lee Silverman em 3 pacientes com parkinsonismo atípico, o paciente com AMS é descrito apresentando hipofonia importante, estridor respiratório durante o sono, tremor de língua, redução da força da língua, diminuição da coordenação do músculo orbicular dos lábios, loudness reduzida, monoloudness, soprosidade extrema, pitch grave, monotom, voz rouca, com redução da expressividade e da precisão articulatória, e velocidade de fala aumentada. Os estudos acústicos com pacientes com atrofia olivopontocerebelar apontam diadococinesia fonoarticulatória alterada ${ }^{24,25}$.

$\mathrm{Na}$ avaliação laringológica de pacientes com a síndrome de Shy-Drager, Duffy (1995) e Swan et al (1999) relatam a presença de estridor laríngeo e sugerem que esta manifestação seja resultante de paralisia uni ou bilateral das pregas vocais.

O estudo de Perez et al (1996) $)^{26}$ analisou 22 Pacientes com doença de Parkinson e 7 pacientes com ParkinsonPlus (PP) e encontraram que $55 \%$ dos pacientes com DP apresentaram tremor predominantemente laríngeo, enquanto $65 \%$ dos pacientes com PP apresentaram tremor predominantemente nas cartilagens aritenóideas.

$\mathrm{O}$ único paciente, descrito sob o diagnóstico de AMS, que foi avaliado laringologicamente, é citado por Countryman et al (1994) $)^{23}$ apresentando hiperemia das pregas vocais, excesso de secreção, tremor, arqueamento de pregas, diminuição da onda mucosa e irregularidade na movimentação da pregas vocais.

Considerando os aspectos citados, verificamos que 
embora muitos estudos apontem a importância da disartrofonia na atrofia de múltiplos sistemas, ainda são poucos aqueles que caracterizam perceptualmente a alteração na comunicação oral nestes pacientes, e, raros os que oferecem dados acústicos e laringológicos desta entidade. Sugerimos que mais estudos se proponham a aprofundar o conhecimento fonoaudiológico destes pacientes afim de desenvolvermos um método terapêutico que melhore a qualidade de vida do paciente com AMS.

\section{REFERÊNCIAS BIBLIOGRÁFICAS}

1. Déjérine, J. \& Thomas, A.A - L'atrophie olivo-ponto-cérébelleuse. Nouv Iconog Salpêtrière 1900; 13: 330 - 370.

2. Shy G.M. \& Drager, G.A. - A neurological syndrome associated with orthostatic hypotension. Arcg Neurol 1960; 2: 511 $-527$.

3. Graham J.G. \& Oppenheimer D.R. - Orthostatic hypotension and nicotine sensitivity in a case of multiple system atrophy. J Neurol Neurosurg Psychiatry 1969;32(1):28-34.

4. Siemers E. Multiple System Atrophy. Med Clin N Am 1999; 83: 381-392.

5. Hanna P A, Jankovic J, Kirkpatrick J B. Multiple System Atrophy: The putative causative role of environmental toxins. Arch Neurol 1999; 56: 90-94.

6. Litvan I, Goetz C G, Jankovic J, Wenning GK, Booth $\vee$ et al. What is the accuracy of the clinical diagnosis of Multiple System Atrophy: A clinicopathologic study. Arch Neurol 1997; 54: 937-944.

7. Wenning, G.K.; Ben-Shlomo, Y.; Hughes, A.; Daniel,S.E.; Less, A.; Quinn,N.P. - What clinical features are most useful to distinguish definite multiple system atrophy from Parkinson's disease?. J Neurol Neuros Psych 2000; 68 (4): 434-440.

8. Quinn N - Multiple system atrophy - the nature of the beast. J Neurol Neurosurg Psychiatry 1989; 52 : 78-89.

9. Bassich CJ, Ludlow CL, Polinsky RJ - Speech symptoms associated with early sings of Shy-Drager syndrome. J Neurol Neurosurg Psychiatry 1984; 47: 995-100.

10. Swan, L. \& Dupont, J. - Multiple system atrophy. Phys Ther 1999; 79: 488-494.

11. International Medical Workshop Covering Progressive Supranuclear Palsy, Multiple System Atrophy, and Cortico Basal Degeneration. Mov Disord 2001; 16: 382-395.

12. Knopp, D.B.; Ferraz, H.B.; Barsottini, O.G.P. - Avaliação Fonoaudiológica na Atrofia de Múltiplos Sistemas (AMS): Estudo com 5 pacientes. Arch NeuroPsiQuiatr 2002b; 60(3-A): 19-23.

13. Ramig, L.O. \& Sherer, R. - Speech therapy for neurological disorders of the larynx. IN: Blitzer, A.; Sazaki, C.; Fahn, S.; Brin, M. \& Harris, K. (eds.) - Neurological disorders of the larynx. New York, Thieme, 1992. p.248-78.

14. Carrara-De Angelis, EC - Deglutição, configuração laríngea, análise clínica e acústica computadorizada da voz de pacientes com Doença de Parkinson [tese]. São Paulo: Universidade Federal de São Paulo; 2000.

15. Darley FL, Aronson A, Brown JR- Differential diagnostic patterns of dysarthria. J Speech Hear Res 1969a; 12: 246252.

16. Darley FL, Aronson A, Brown JR - Clusters of deviant speech dimensions in disarthrias. J Speech Hear Res 1969b; 12: $462-468$.

17. Otoxby M - Parkinson's disease patients and their social needs. London: Parkinson's disease Society, 1982.

18. Streifer M, Hofman S - Disorders of verbal expression in Parkinsonism, In Hassler RG \& Christ JF (Eds) - Adv Neurol 1984; 40: 385-393.

19. Wenning, G.K.; Ben-Shlomo, Y.; Magalhães, M.; Daniel, S.E.; Quinn, N.P. - Clinical features and natural history of multiple system atrophy - An analysis of 100 cases. Brain 1994; 117: 835-845.

20. Kluin KJ, Gilman S, Lohman M, Junck L - Characteristics of the dysarthria of multiple system atrophy. Arch Neurol 1996; 53: 545-8.

21. Rehman HU - Multiple system atrophy. Postgrad Med J 2001; 77: 379-382.

22. Duffy JR - Motor speech disorders: substrates, differential diagnosis and management. St. Louis, Mosby, 1995.

23. Countryman S, Ramig LO, Pawlas, AA - Speech and voice deficits in parkinsonian plus syndromes: Can they be treated? J Med Speech Lang Pathol 1994; 2: 211-25.

24. Gilman, S. \& Kluin, D. - Perceptual analysis of speech disorders in Friedreich disease and olivopontocerebellar atrophy. IN: Bloedel J.R. (ed.) - Cerebellar functions, New York, Springer - Verlag, 1984.

25. Hartman D.E. \& O'neil B.P. - Progressive disfluency, dysphagia, dysarthria: a case of olivopontocerebellar atrophy. IN: Yorkston, K.M. \& Beukelman, D.R. (eds.) - Recent advances in dysarthria, Boston, College-Hill, 1989.

26. Perez, K.S.; Ramig, L.O.; Smith, M.E., Dromey, C. - The Parkinson larynx: tremor and videostrobocopic findings. J Voice 1996; 10 (4): 354-61. 